\title{
ANÁLISIS DENDROCLIMATOLÓGICO (CASTANEA SATIVA MILL) EN EL MACIZO DEL MONTSENY²
}

\author{
por \\ Ricardo Génova ${ }^{1}$ y Carlos A. Gracia ${ }^{1}$
}

\section{SUMMARY}

The importance of the variable «hours of sunlight» (as an estimator of irradiance) in the growth processes of the Chestnut (Castanea sativa) has been pointed out.

Mild temperatures and high precipitation in the months preceding the vegetative period have a negative effect on the ring width increment. This seems to agree with the hypothesis that considers the tree's dormancy period as a security factor against climatic events.

The August precipitation has a positive effect on the ring width increment. This comes about because there is no pause in either metabolic activity or growth rate due to water stress.

\section{INTRODUCCION}

La dependencia existente entre la climatología, globalmente considerada y la producción de una especie vegetal resulta evidente. No lo es tanto, la forma en que actúa sobre el crecimiento de la planta cada factor climático por separado y si consideramos que cada uno de ellos, puede afectar favorablemente en una época del año y desfavorablemente en las otras, llegamos a un esquema de gran complejidad del que apenas disponemos de datos.

El crecimiento anual de los árboles, datado en la formación de los anillos de crecimiento, constituye un registro extraordinario, que permite obtener información acerca de las respuestas de cada aŕbol a los factores climatológicos. Los estudios en dendroclimatología, se han enfocado tradicionalmente hacia la reconstrucción de episodios climáticos del pasado (DOUGLAS 1921, SCHULMAN 1956, H. C. FRITTS 1965, BAILLIE $1973, \ldots$ ) y más raramente para obtener información sobre la respuesta fisiológica de los árboles a los diferentes factores climáticos. En España los intentos de CREUS \& PUIGDEFABREGAS en 1976 y de TOMAS en 1982 son los únicos antecedentes de análisis dendroclimatológico.

(1) Departamento de Ecología. Facultad de Biología. Universidad de Barcelona.

(2) Este trabajo se beneficiado de una ayuda a la Investigación (año 1982) de la Comissió Interdepartamental de Reserca i Innovació Tecnológica (CIRIT) de la Generalitat de Catalunya. 


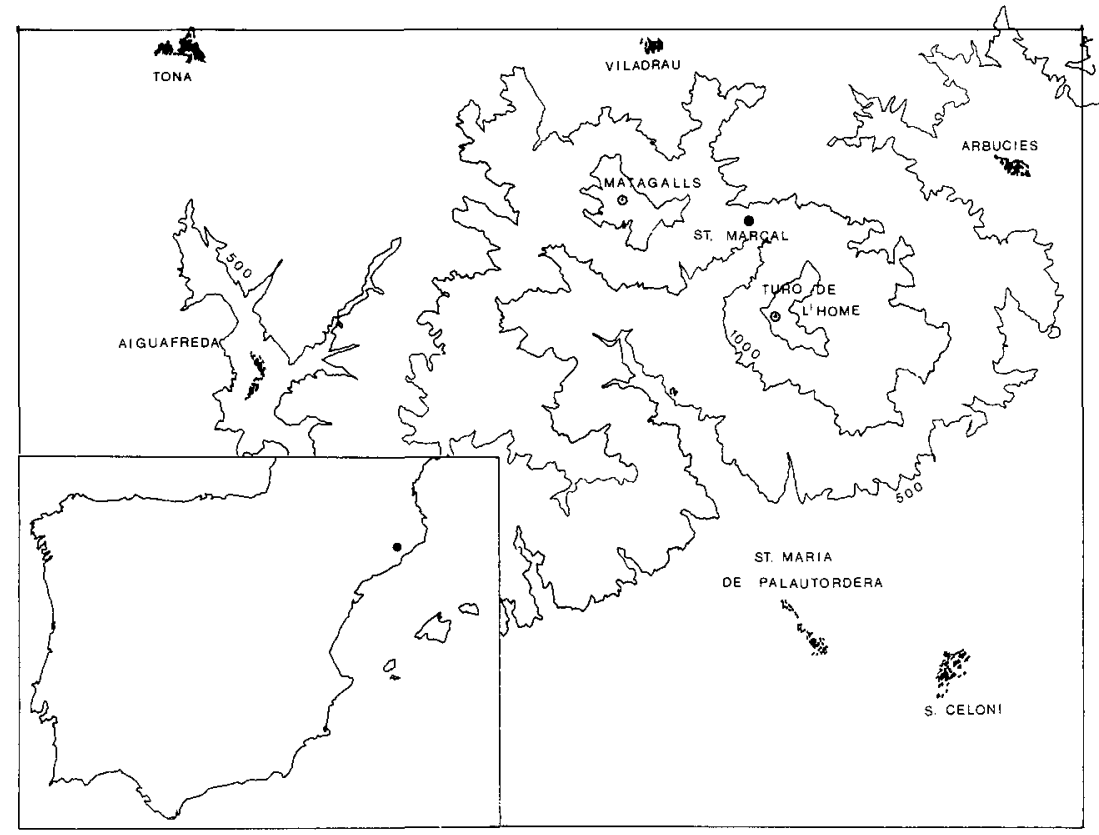

Figura 1.- Localización geográfica del Macizo del Montseny y del área de estudio de Sant Marçal.

El trabajo que aquí se expone representa un intento de interpretación de la respuesta del castaño frente a diversos factores climáticos en el Montseny.

El macizo del Montseny situado en el NE de España, constituye un área geográfica con un interés de estudio singular desde muchos puntos de vista. Numerosas monografías describen estos aspectos, por lo que no insistiremos sobre ellos.

Desde el punto de vista climatológico, el Montseny dispone de un observatorio de primera categoría, situado en la cima del Turó de L'Home, punto culminante del macizo a 1712 metros de altitud de donde proceden los registros climáticos utilizados en el trabajo.

El castaño fue introducido en este área hace varios siglos y se da principalmente en las umbrías silíceas, donde se explota por su rápido crecimiento y tronco recto, con un turno de tala de veinte a veinticinco años, tiempo que tardan en alcanzar el tamaño adecuado para su uso industrial. 
En el momento de la corta, estos bosques presentan una densidad de 2.100 pies por ha. y una biomasa de madera de $239.085 \mathrm{Tm} / \mathrm{ha}$.

Estas características limitan la longitud de la serie de que hemos dispuesto a veinticinco años. Por tratarse de una plantación, los diámetros y la edad de los árboles presentan una uniformidad notable que minimiza el número de muestras necesario para registrar la variabilidad de respuesta de los árboles al clima.

\section{MATERIAL Y METODOS}

En una parcela del castañar de Sant Marçal, se eligieron cinco árboles de la clase diamétrica superior $(16-21 \mathrm{~cm}$.), de modo que constituyeran un grupo lo más homogéneo posible, a fin de eliminar la varianza debida a factores edafológicos o topográficos.

De cada árbol se extrajeron dos «cores» con la barrena de Pressler, a la altura de 1,30 m. y de orientaciones opuestas (norte y sur), al objeto de registrar las diferencias en el espesor de los anillos debidas a la orientación en el tronco.

Los «cores» se dejaron secar al aire, montados sobre estructuras que impiden su deformación. Una vez secos se pulieron y se tiñieron con una solución de cloruro de zinc iodado, que facilita la lectura, al resaltar las diferencias existentes entre la madera temprana, formada en primavera, con células grandes y de menor densidad y la madera tardía, de formación otoñal, con células más pequeñas y de mayor densidad. Las lecturas se realizaron con lupa binocular. En ninguna de las muestras se presentaron anomalías de crecimiento en forma de anillos falsos o ausentes.

La serie cronológica de cada árbol se estableció promediando los dos «cores» extraídos del mismo. Estas series no son estacionarias, sino que presentan una tendencia debida principalmente a factores internos del árbol relacionados con la edad. Para filtar esta tendencia, a fin de resaltar el efecto de los factores externos, cada serie se ajustó a una función de la forma:

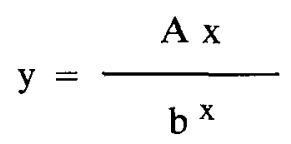




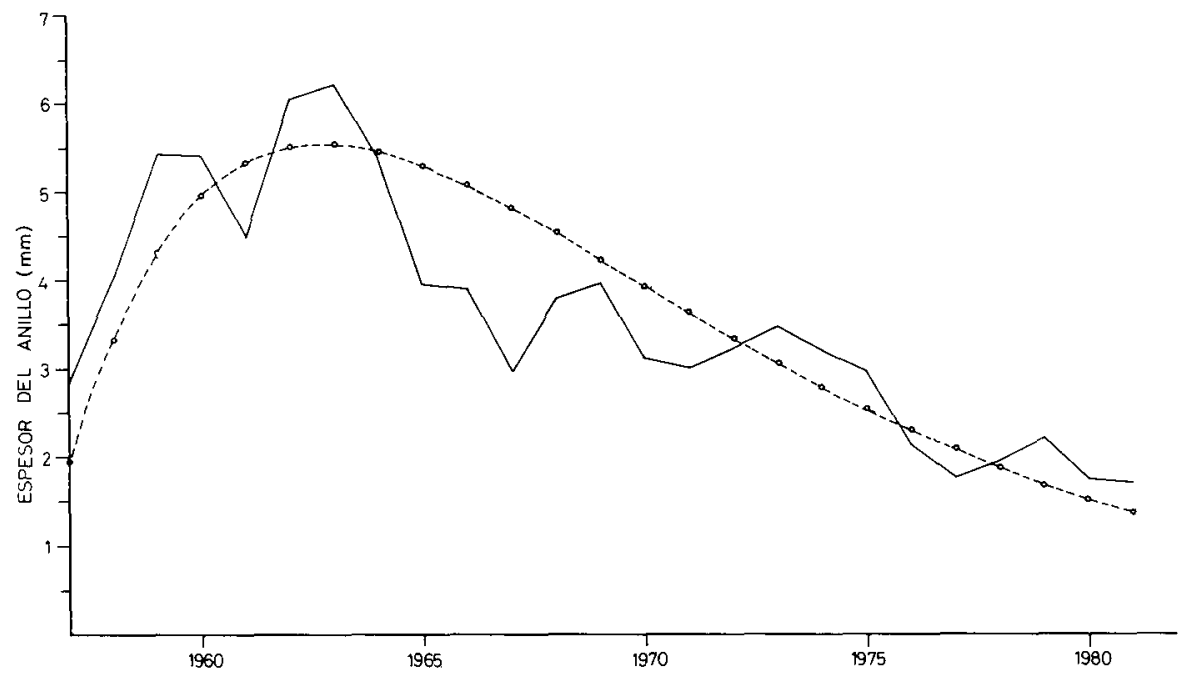

Figura 2." Serie dendrocronológica del core medio de todas las muestras y valores estimados por la función de ajuste utilizada.

que presenta la ventaja de recojer fielmente las variaciones en el crecimiento durante los primeros años, aspecto muy importante tratándose de series cortas. (Fig. 2).

Una vez aplicada la función de ajuste, ponderamos los incrementos anuales, transformándolos en índices:

$$
I=\frac{X(\text { real })}{X(\text { estimado })}
$$

siendo X el ancho del anillo. Esta transformación se realiza con el fin de homogeneizar la varianza de todas las series y situar la media en torno a un valor cercano a 1 .

A continuación se calculó el coeficiente de sensibilidad medio (SM), definido como media porcentual de cambio de cada medida del ancho del anillo con respecto al próximo (DOUGLASS, 1936). 


$$
S M=\frac{1}{n-1} \sum_{t=1}^{t=n-1} \frac{2\left(I_{t}+1-I_{t}\right)}{I_{t+1}+I_{t}}
$$

donde $I_{t}$ son los índices del ancho de los anillos en el año $t, I_{t}+1$ son los índices del ancho de los anillos en el año siguiente y $\mathrm{n}$ es el número de años que presenta la serie. Este coeficiente se puede calcular también para el ancho del anillo real. En el caso de calcularlo con los índices este coeficiente puede variar entre 0 , donde no existe diferencia entre los anchos de los anillos, y 2 donde valores vecinos pueden presentar las máximas diferencias. (FRITTS, 1976).

Los valores de los coeficientes de sensibilidad tanto para el ancho de los anillos como para los índices se sitúan en torno a valores de 0,16 (Tabla I).

El coeficiente de interdatación se calculó como el coeficiente obtenido entre el promedio de los SM de todos los cores y la SM de la serie final promediada. Cuando este coeficiente es mayor a $1 / \mathrm{n}$, siendo n el número de series utilizadas, se considera que dichas series no son aleatorias sino que tienden a variar juntas (SCHULMAN, 1956).

\section{Análisis de las variables climáticas.}

Los datos climatológicos se recogieron del observatorio del Turó de L'Home situado a una distancia de unos $6 \mathrm{Km}$. de la zona estudiada.

Las variables recogidas y posteriormente analizadas fueron: horas de sol mensuales, precipitación mensual, media mensual de las temperaturas máximas, media de las temperaturas mínimas y media de las temperaturas medias en los doce meses del año, por lo que se partía de un análisis con sesenta variables. El número de años (casos) estuvo limitado por las horas de sol de las que sólo se disponía registro de los últimos 21 años.

$\mathrm{Al}$ ser estas variables iniciales muy redundantes en información, el trabajo se inició seleccionando un menor número de variables que recogiesen la máxima información. 
$\frac{0}{\square}$

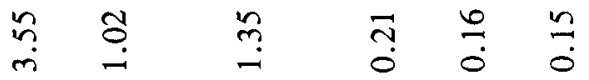

당

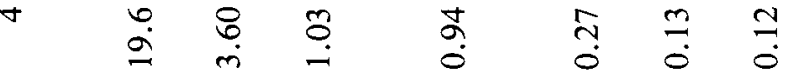

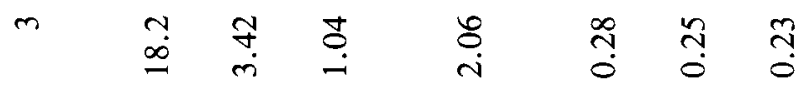

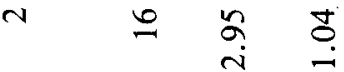

$\stackrel{\circ}{0} \stackrel{1}{0}$

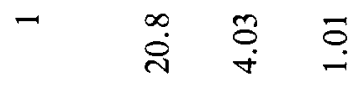

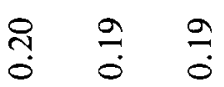

छ

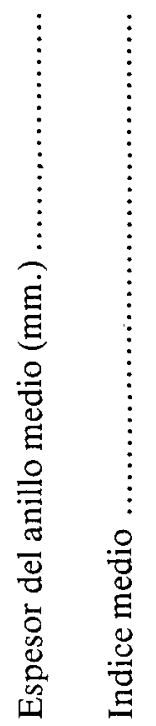

$\frac{1}{2}$

$\frac{\infty}{0}$

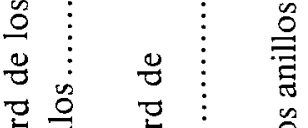


Esta selección se realizó en base a un análisis de componentes principales. En este análisis podemos averiguar el círculo de contribución equilibrada, como referencia para juzgar la contribución de cada descriptor en una proyección dada de espacio reducido (LEGENDRE \& LEGENDRE, 1979). Las variables que quedan fuera de este círculo de contribución equilibrada, poseen una información en el análisis superior a la información media, son por tanto las que poseen mayor significación y contribuyen a explicar la mayor parte de la varianza.

\section{Análisis dendroclimatológico}

La formación de los anillos anuales es el resultado de la interacción de un elevado número de variables. Resulta evidente por tanto, que las correlaciones entre cada variable tomada aisladamente y el crecimiento anual del árbol presente valores bajos. Es en la relación entre todas estas variables interactuando en conjunto donde hay que buscar la información que permitirá determinar qué factores son los que mayor influencia poseen sobre el incremento anual de madera.

Un análisis de regresión múltiple paso a paso, fue utilizado para cuantificar la relación entre las treinta y cuatro variables seleccionadas por componentes principales y la variación del espesor de los anillos de la serie promediada. Otros análisis realizados, mostraron la importancia de incluir la variable «horas de sol mensuales» en el análisis para lograr una buena predicción de la serie estudiada. Este análisis permite valorar el peso de cada una de las variables en la adecuación de predicción, así como los efectos de sinergia que pudieran derivarse con la inclusión de dos o más variables actuando a la vez.

\section{RESULTADOS}

En la tabla 1 se resumen los parámetros estadísticos sobre las series. Los valores de los coeficientes medios de sensibilidad (SM) son bajos, debido a las condiciones climáticas imperantes en la zona que son bastante suaves. La posible acentuación del clima en invierno o verano queda tamponada por efecto de las nieblas muy frecuentes que se forman en esta zona, 191 días al año (PANAREDA, 1973). En ambientes extremos, donde los factores climáticos son limitantes, otros autores encuentran valores más altos; en zonas semidesérticas el índice se sitúa en torno a 0.7 (H. C. 


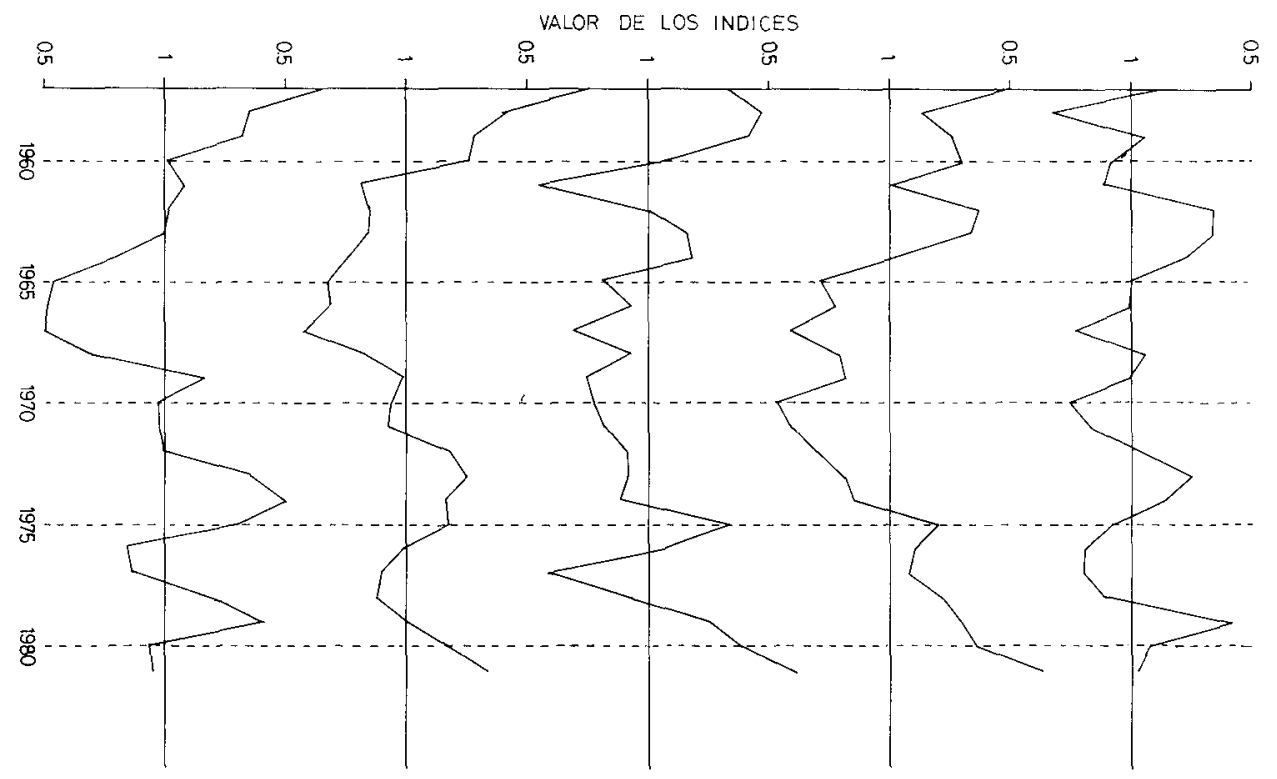

Figura 3.- Cronologías del espesor de los anillos, expresados como indices, para los árboles muestreados.

FRITTS et al. 1965) y en alta montaña en torno a 0.3 (CREUS \& PUIGDEFABREGAS, 1976).

Cabe resaltar el caso del árbol 3 que presentó un SM más alto de 0.25 . La razón de esta diferencia debe buscarse en otras causas no climáticas, que afectan al crecimiento anual. Este árbol presentaba otros pies de castaño muy próximos, con distancias de 0.40 y 0.46 metros, mientras que en el resto de los árboles estudiados las distancias a sus vecinos eran superiores a dos metros. Esta característica hace pensar que el factor de competencia refuerza el efecto negativo sobre el incremento anual de madera cuando las condiciones climáticas son más desfavorables, efecto que se refleja en el coeficiente medio de sensibilidad. 
El coeficiente de interdatación nos muestra que las series de los árboles estudiados tienen una pauta de variación común en todas ellas.

El análisis en componentes principales permitió estudiar la contribución de cada variable en la definición del año climático.

Los tres primeros factores de este análisis explican un $52 \%$ de la varianza total (Fig. 4).

El primer factor explica un $20 \%$ de la varianza, se interpretó como estacionalidad anual, ya que permite diferenciar claramente los grupos compuestos por meses invernales (diciembre, febrero y marzo) los primaverales-otoñales (mayo, junio y septiembre) y los veraniegos (julio y agosto).

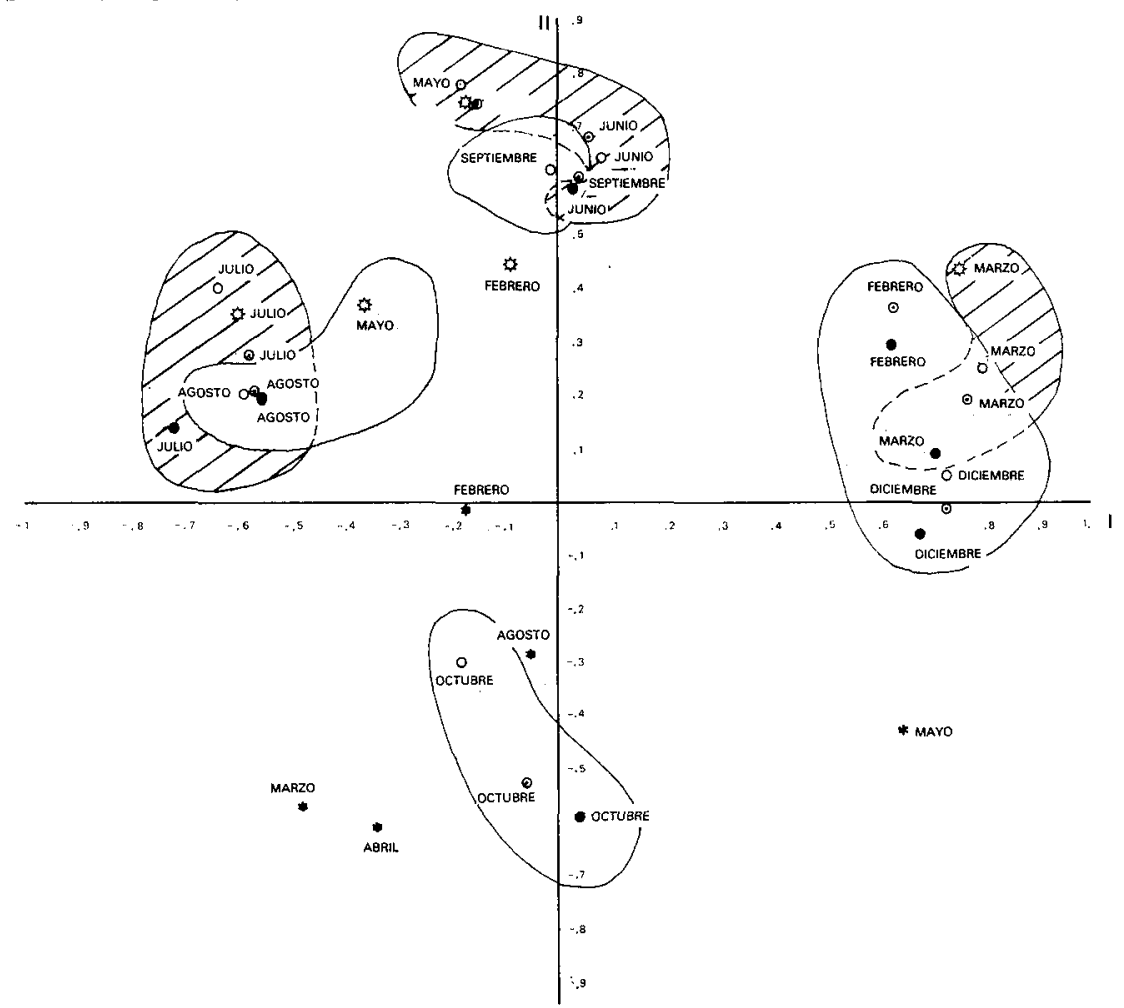

Figura 4.- Análisis en componentes principales: representación espacial del conjunto de las variables en los dos primeros factores. Sólo se representan las 34 variables que contribuyen mejor a definir el año climático.

Se diferencian los grupos separados por el tercer factor: en blanco los que presentan cargas positivas y en rayado los que presentan factores de carga más bajos o negativos.

Símbolos utilizados: * Horas de sol, * Precipitación total, $\bigcirc$ Temperatura media de las máximas,

- Temperatura media de las minimas, $\odot$ Temperatura media de las medias. 


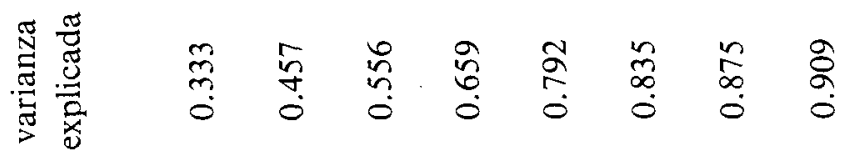

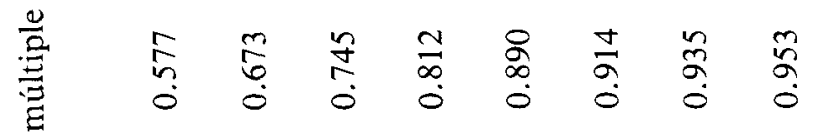

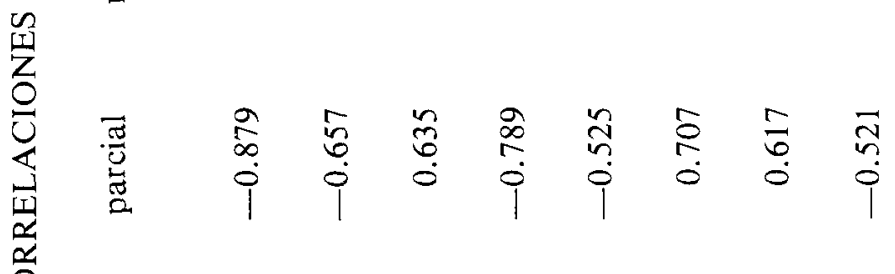

蕞

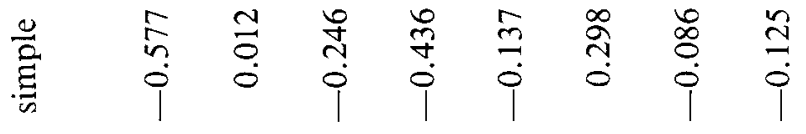

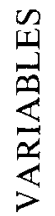

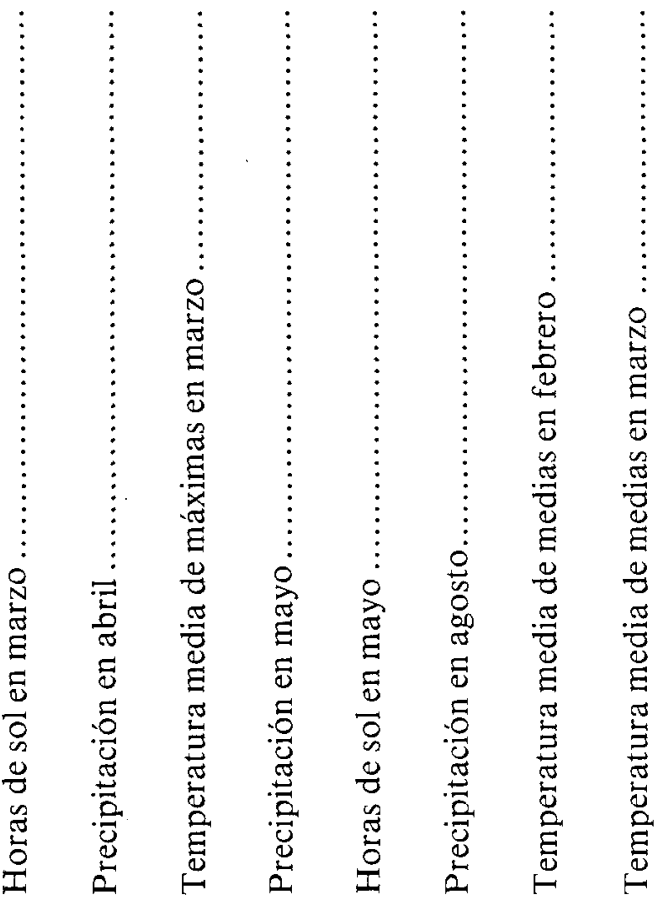


El segundo factor explica un $17 \%$ de la varianza y se interpretó como un eje asociado con las precipitaciones, ya que diferencia las variables de precipitación total mensual del resto de las variables: horas de sol y medias de las temperaturas.

El tercer factor explica un $15 \%$ de la varianza y se interpreta como estacionalidad dentro de los grupos anteriormente establecidos, ya que separa los meses primaverales (mayo y junio) de los otoñales (septiembre y octubre), así como los meses más fríos del invierno (diciembre y febrero) del más templado (marzo). En el grupo constituído por los meses veraniegos tiende a separar julio y agosto.

Estè análisis realizado sobre las variables climáticas permitió definir el año en función de 34 variables. En este conjunto de variables se incluyeron todas aquellas que explicaron una parte de la varianza superior a la varianza media, reflejado por el círculo de contribución equilibrada.

El análisis de regresión múltiple paso a paso permitió seleccionar ocho variables ordenadas según contribución en la predicción del espesor de los anillos. Las ocho variables explican en conjunto el $90 \%$ de la varianza de la serie de los índices.

Las correlaciones simples entre estas ocho variables y la serie de los índices del core medio, así como las correlaciones parciales y el incremento en el cuadrado de la correlación múltiple, varianza explicada, queda reflejado en la tabla 2.

La ecuación de predicción resulta ser:

$$
\begin{aligned}
y= & 1.575-0.047 x_{1}-0.007 x_{2}+0.175 X_{3}-0.015 X_{4} \\
& -0.012 x_{5}+0.012 x_{6}+0.027 X_{7}-0.125 X_{8}
\end{aligned}
$$

$\mathrm{X}_{1}$ horas de sol en marzo.

$\mathrm{X}_{2}$ precipitación total en abril.

$\mathrm{X}_{3}$ temperatura media de las máximas en marzo.

$\mathrm{X}_{4}$ precipitación total en mayo.

$\mathrm{X}_{5}$ horas de sol en mayo.

$\mathrm{X}_{6}$ precipitación total en agosto.

$\mathrm{X}_{7}$ temperatura media de las medias en febrero.

$\mathrm{X}_{8}$ temperatura media de las medias en marzo. 


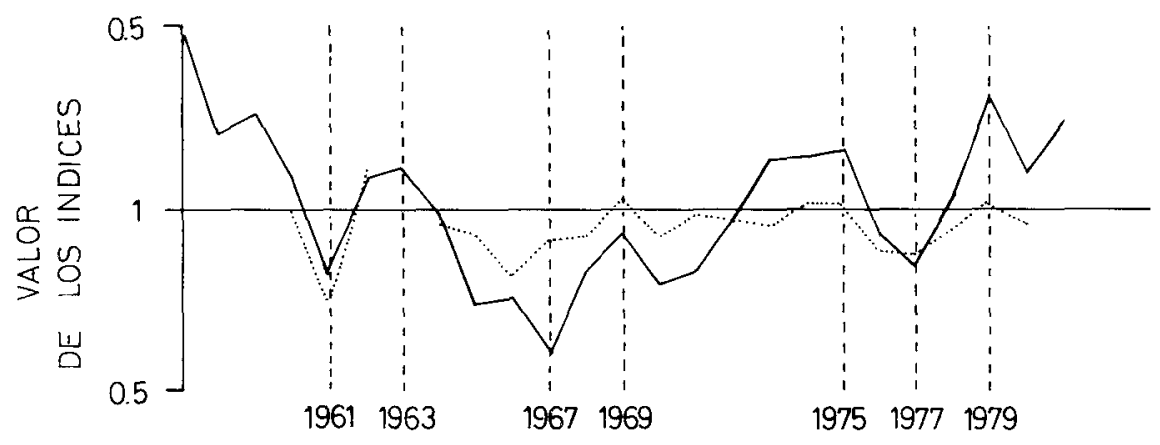

Figura 5.- Cronologia del espesor de los anillos del core medio, expresados en indices (línea continua) y función de predicción del espesor de los anillos en función de las ocho variables seleccionadas por el análisis (línea de puntos). Se señalan los años que presentan máximos o mínimos singulares en el espesor de los anillos.

En la figura 5 se representan los índices estimados a partir de la ecuación de predicción.

\section{CONCLUSIONES}

Las horas de sol destacan como el parámetro con mayor influencia sobre el crecimiento anual de los castaños. De éstas las horas de sol en marzo, resultan ser las más importantes con una contribución del 33,28\% (a la varianza del crecimiento).

En la tabla 3 se resumen los valores de las 8 variables seleccionadas, en años en los que el índice del espesor de los anillos alcanzó máximos o mínimos singulares.

Si consideramos los años con escaso crecimiento (1961, 1967 y 1977) (Fig. 5) comprobamos que las horas de sol en marzo destacan por presentar valores elevados, que influyen negativamente sobre el crecimiento, en tanto que en los años con mayor crecimiento los valores de esta variable fueron más bajos (1963, 1969, 1975 y 1979) (Fig. 5).

Las temperaturas medias de las máximas en marzo, tienen un efecto semejante por tratarse de una variable estrechamente correlacionada con la insolación.

La precipitación total en mayo, presenta una influencia negativa 


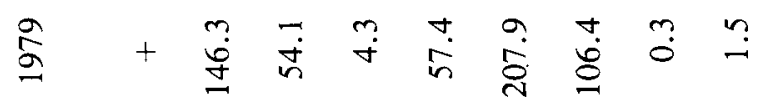

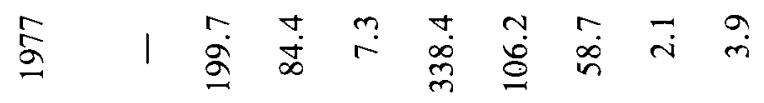

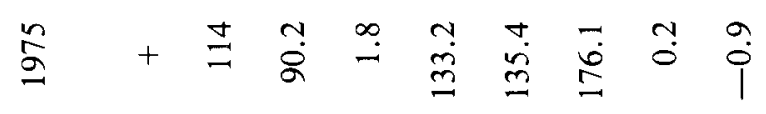

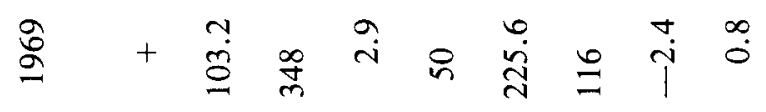

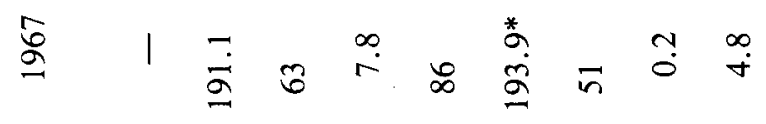

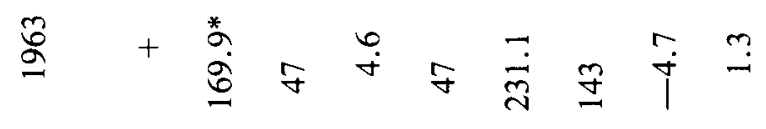

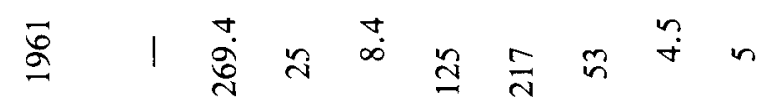


sobre el espesor de los anillos. No se corresponde el valor de 1975, donde con un valor alto, la influencia sobre el crecimiento es favorable. Probablemente sea debido al efecto tan negativo sobre el crecimiento que presentaron en este año las variables horas de sol en marzo y temperatura media de las máximas del mismo mes, variables éstas que tienen el mayor peso en la predicción de la variable dependiente.

La precipitación total en agosto es la única variable del conjunto que posee un efecto positivo sobre el crecimiento anual del castaño. Los valores más altos (por encima de $100 \mathrm{ml}$. de precipitación) se corresponden con los años de crecimientos máximos singulares.

Para el caso de las temperaturas medias de medias en los meses de febrero y marzo el esquema se mantiene, correspondiéndose valores bajos en el crecimiento con los máximos de estas variables y altos en el crecimiento con los mínimos.

El hecho de que en los meses previos al período de actividad vegetativa, suaves temperaturas y elevadas precipitaciones tengan un efecto negativo sobre el incremento en el espesor de los anillos, redunda sobre la hipótesis de que el período de latencia es para los árboles un «factor de seguridad» frente al clima.

Cuando la latencia queda rota, el clima afecta directamente la producción de hormonas reguladoras del crecimiento e influye sobre los procesos de crecimiento como son la fotosíntesis, la acumulación de sustancias nutritivas y el balance hídrico en el árbol.

Este período de latencia es importante que no cese en un momento (final) del invierno, cuando por efecto de altas temperaturas pudieran comenzar a desarrollarse los brotes jóvenes, que se verían dañados por eventuales heladas tardías. El tejido del cambium en las ramas es especialmente susceptible de sufrir daño por esta causa.

En una especie caducifolia, esta característica condiciona a la planta sobre el momento de iniciar su actividad funcional. Estos condicionantes deben haber modelado la población de castaños desde su implantación en el Montseny, seleccionando los individuos que rompen el período de latencia cuando no exista posibilidad de verse dañados por heladas tardías como las que se dan en el período inicial de la primavera en los climas de montaña. 
Más evidente resulta la importancia del mes de agosto. El crecimiento de los árboles responde a un sistema pulsante en el que además de la latencia permanente, típica del invierno, se dan períodos de escasa o nula actividad con latencia temporal de pocos días o semanas. Este es el caso de inactividad que presentan muchas especies forestales durante el verano, cuando la sequía estival se traduce en un fuerte déficit hídrico que paraliza la producción.

Cuando agosto se presenta lluvioso, estas condiciones no se producen y el árbol no ve paralizado su crecimiento, lo que se traduce en un mayor incremento de madera anual y por tanto un mayor incremento en el espesor de los anillos del año en cuestión.

Agradecimientos.

Al Dr. Margalef que propuso este apasionante tema de estudio.

A Xavier Millet por sus continuos desvelos en el tratamiento estadístico de los datos.

A las personas del Departamento de Ecología que con sus discusiones ayudaron en la elaboración final del escrito.

A todos ellos nuestro sincero agradecimiento.

\section{BIBLIOGRAFIA}

BAILLIE, M. G. L., (1972). Tree ring dating and archaeology. Croom Helm, London.

CREUS, J. y PUIGDEFABREGAS, J., (1976). Cilmatología histórica y dendrocronología de Pinus uncinata (Ramond). Cuadernos de investigación, 2 (2): 17-30. Logroño.

DOUGLASS, A. E., (1936). Climatic cycles and tree growth. Vol. III, A study of cycles. Carnegie Inst. Wash. Publ. 289.

FRITTS, H. C., (1974). Relationships of ring widths in Arid Site conifers to variations in monthly temperature and precipitation. Ecol. Monogr. 44 (4): 411-440.

FRITTS, H. C., (1976). "Tree rings and climate». Academic Press, London. 
FRITTS, H. C., SMITH, D. G., CARDIS, J. W., and BUDELSKY, C. A., (1965). Tree ring characteristics along a vegetation gradient in nothern Arizona. Ecology 46, 393-401.

LEGENDRE, L. \& LEGENDRE, P., (1979). Ecologie numerique. Ed. Masson, París.

PANAREDA i CLIPES, J. M., (1973). Itinerari geografic al Montseny. Universitat de Barcelona. Departamento de Geografía.

SCHULMAN, E., (1956). Dendroclimatic changes in semiarid America, Tucson, $142 \mathrm{pp}$.

TOMAS i MARTORELL, C., (1982). Estudi dendrometric de la producció de faig a la vall de Santa $\mathrm{Fe}$ del Montseny. Tesis de licenciatura. 